\title{
Prenatal Stress Induces Skeletal Malformations in Mouse Embryos
}

\author{
Jongsoo Kim ${ }^{1}$, Hyo Jung Yun ${ }^{1,2}$, Ji-Yeon Lee ${ }^{1, \uparrow}$ and Myoung Hee Kim ${ }^{1,2, \uparrow}$ \\ ${ }^{1}$ Department of Anatomy, Embryology Laboratory, ${ }^{2}$ Brain Korea 21 Plus Project for Medical Science, \\ Yonsei University College of Medicine, Seoul 120-752, Korea
}

\begin{abstract}
Dexamethasone, a synthetic glucocorticoid (GC), is clinically administered to woman at risk for premature labor to induce fetal lung maturation. However, exposure to repeated or excess GCs leads to intrauterine growth restriction (IUGR) and subsequently increases risk of psychiatric and cardio-metabolic diseases in later life through fetal programming mechanisms. GCs are key mediators of stress responses, therefore, maternal nutrient restriction or psychological stress during pregnancy also causes negative impacts on birth and neurodevelopment outcome of fetuses, and other congenital defects, such as craniofacial and skeletal abnormalities. In this study, to examine the effect of prenatal stress on fetal skeletal development, dexamethasone (1 mg/kg [DEX1] or $10 \mathrm{mg} / \mathrm{kg}$ [DEX10] maternal body weight per day) was administered intraperitoneally at gestational day 7.5 9.5 and the skeletons were prepared from embryos at day 18.5. Seven out of eighteen (39\%) embryos treated with DEX10 showed axial skeletal abnormalities in either the T13 or L1 vertebrae. In addition, examination of the sternum revealed that xiphoid process, the protrusive triangular part of the lower end of the sternum, was bent more outward or inward in DEX group embryos. In conclusion, our findings suggest a possible link to the understanding of the effect of uterine environment to the fetal skeletal features.
\end{abstract}

Key Words: Dexamethasone, Prenatal stress, Embryo development; Axial skeleton

\section{INTRODUCTION}

Glucocorticoids (GCs) are involved in normal developmental processes of diverse organs such as kidneys, lung, heart, pancreas, and gut. As GC has multiple metabolic and immunological effects, it is usually applied to treat patients with inflammatory, allergic, and immune disorders in children and adults. Although it has many positive effects

\footnotetext{
* Received: October 21, 2014 / Revised: December 5, 2014

Accepted: December 5, 2014

${ }^{\dagger}$ Corresponding author: Ji-Yeon Lee. Department of Anatomy, Embryology Laboratory, Yonsei University College of Medicine, Seoul 120-752, Korea. Tel: +82-2-2228-1657, Fax: +82-2-365-0700

e-mail: jylee517@yuhs.ac

${ }^{\dagger}$ Corresponding author: Myoung Hee Kim. Department of Anatomy, Embryology Laboratory, Brain Korea 21 Plus Project for Medical Science, Yonsei University College of Medicine, Seoul 120-752, Korea.

Tel: +82-2-2228-1647, Fax: +82-2-365-0700

e-mail: mhkim1@yuhs.ac

(C) The Korean Society for Biomedical Laboratory Sciences. All rights reserved.
}

with drug use, patients taking chronic GC therapy often suffer from potential side effects, in particular on the skeleton, including growth retardation during childhood and decreased bone quality in adults (Canalis 2005; Henneicke et al., 2014). During pregnancy, GC is clinically administered to pregnant woman at risk for premature labor to induce fetal lung maturation (Ward 1994; Sloboda et al., 2005). Despite the clear benefits of antenatal GC therapy, exposure to repeated or excess GCs have been known to cause a intrauterine growth restriction and subsequently increased risk of psychiatric and cardio-metabolic diseases in later life (Newnham and Moss 2001). Besides, different types of prenatal stress during pregnancy have been also revealed to cause other congenital defects, such as craniofacial and skeletal anomalies as well as a reduction of birth weight, in a variety of animal models (Śliwa et al., 2006; Lee et al., 2008; Choe et al., 2011).

A few studies have demonstrated the effect of prenatal 
stress and excess glucocorticoid on fetal skeletal development, and on skeletal growth and bone mineralization after birth as a long-term effect (Swolin-Eide et al., 2002; Śliwa et al., 2006; Lee et al., 2008; Choe et al., 2011). However, there is still no coherent connection between a specific type of stressor and the concomitant skeletal changes. Since the prenatal stress affects on different tissues in a variety of different ways, particularly during vulnerable periods of developmental processes, more evidence is needed to determine the relationship between prenatal experiences and the skeletal growth and the bone health in later life.

We have recently demonstrated that administration of dexamethasone (DEX), a synthetic GC, during the gestational day 7.5 9.5 of pregnant mice causes placental defect and embryonic growth restriction in a sex-dependent manner (Lee et al., 2012; Yun et al., 2014). Here, we examined the effect of prenatal DEX treatment on fetal skeletal development on day 18.5. Developmental defects of the axial skeleton found in this model will provide an insight into the long-lasting effect of prenatal GC on the growth and the maturity of the skeleton.

\section{MATERIALS AND METHODS}

\section{Experimental animals and injection of DEX}

All animals were handled according to the guideline for the Care and Use of Laboratory Animals of Yonsei University College of Medicine. The protocol for obtaining embryos was approved by the Committee on Animal Research at Yonsei University College of Medicine. Pregnant ICR mice were ordered from Orient Biology (Sungnam, Korea). DEX administration was performed as previously described (Lee et al., 2012). Briefly, either saline (control group) or dexamethasone 21-phosphate disodium salt (1 mg/ $\mathrm{kg}$ or $10 \mathrm{mg} / \mathrm{kg}$, Sigma-Aldrich, St. Louis, MO, USA) was injected on days 7.5, 8.5 and 9.5 of gestation intraperitoneally.

\section{Embryo extraction and skeleton preparation}

The mice were sacrificed at 10 a.m. on gestational day 18.5 using a carbon dioxide chamber. Embryos were extracted from the womb and the body weight and CRL (Crown-rump length) measured. Each sample's skin and internal organs in the chest cavity and lower trunk were removed after being incubated at $70^{\circ} \mathrm{C}$ for 1 hour. They were fixed in $95 \%$ ethanol for $2 \sim 4$ days and transferred to glass bottles containing acetone and kept overnight. Each sample was washed and stained with $0.03 \%$ Alcian Blue solution (Sigma) overnight. After being put in $95 \%$ ethanol for 3 hours, the embryos were stored in $2 \%$ potassium hydroxide in distilled water (DW) for 24 hours and stained with $0.005 \%$ Alizarin Red solution (Sigma) for 16 hours. The samples were then cleared in $1 \%$ potassium hydroxide in $20 \%$ glycerol for 2 days and transferred to increasingly concentrated glycerol solutions ( $50 \%$ to $80 \%$ glycerol in $95 \%$ ethanol solution for 1 day each).

\section{Microscope observation and identification}

The samples were observed using a light microscope (Olympus SZX10) under varying degrees of brightness and hue. Each spinal column was identified using dorsal and ventral views of the trunk and several anatomical landmarks (T6 and T7 ribs conjoin near the end of the sternum, vertebral columns after caudal 4 lack dorsal processes, etc.). Embryos exposed to DEX were compared to specimens of the control group for abnormalities in the skeletal structure.

\section{RESULTS}

\section{Effect of prenatal DEX treatment on fetal vertebral column}

A total of 36 embryos (18 embryos from 3 mothers with saline, and the same number with $1 \mathrm{mg} / \mathrm{kg}$ of DEX (DEX1) and $10 \mathrm{mg} / \mathrm{kg}$ of DEX (DEX10), respectively) were processed for skeletal preparation at gestational day 18.5. As far as the skeletal system is concerned, all the structures in the vertebral column including cervical, thoracic, lumbar, sacral and caudal were examined and overall no significant difference was observed except on the region between the last thoracic (T13) and the first lumbar (L1) vertebrae (Fig. $1 \mathrm{~A}$ and $\mathrm{B})$. Table 1 summarized the axial skeletal alterations observed in control and DEX group. In our experimental groups, low dose of DEX treatment (DEX1) did not cause any changes in skeletal formation. Contrarily, out of 18 embryos in the DEX10 group, 6 embryos (33\%) showed a 


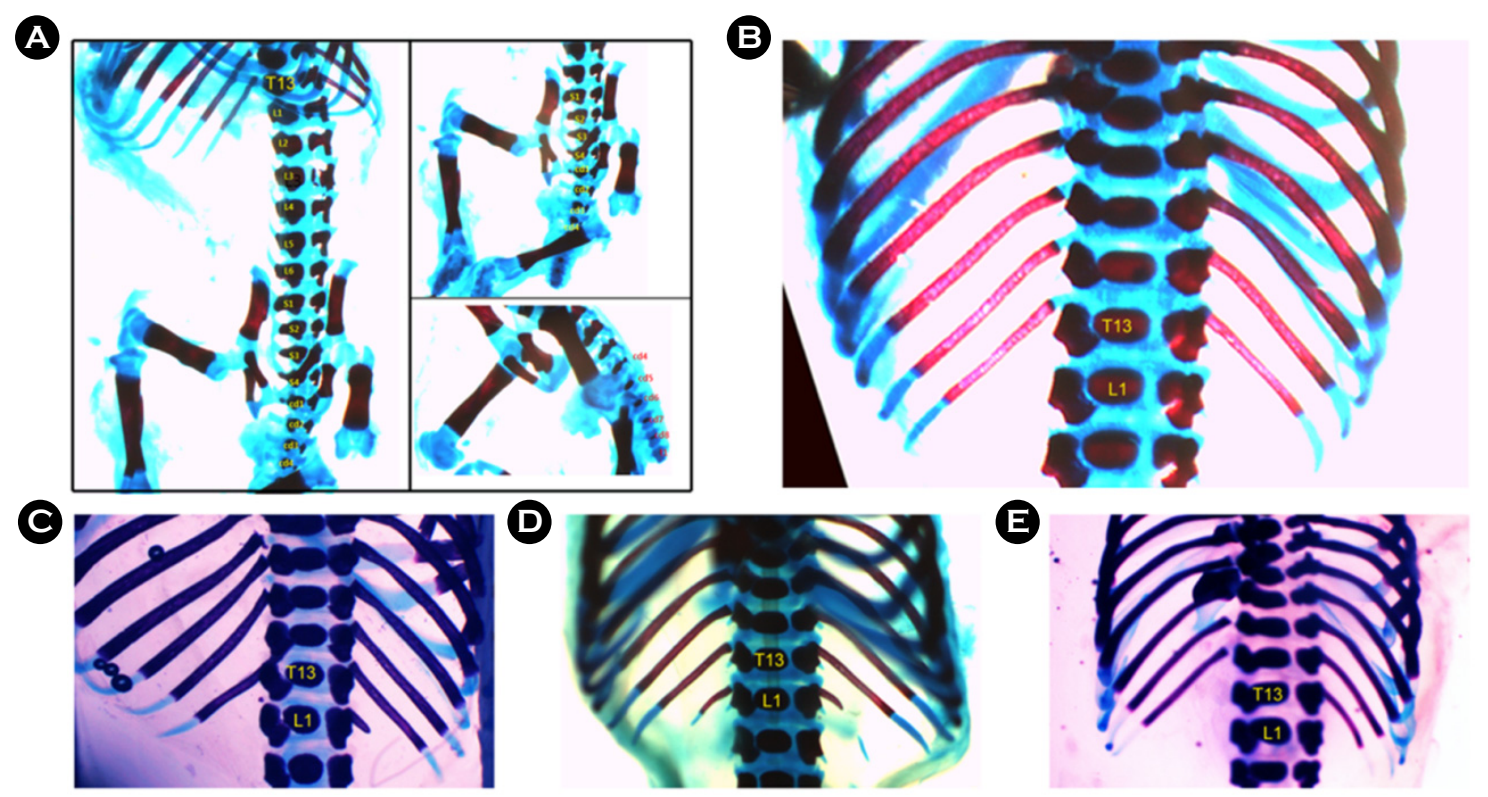

Fig. 1. Axial skeletal abnormalities found in mouse embryos after prenatal exposure to DEX. (A) Overview of skeletons with normal number of thoracic, lumbar, sacral and caudal vertebra. (B) Dorsal view showing normal thoracic vertebrae and rib attachments. (C-D) Anterior transformation of the first lumbar (L1) vertebra in the $13^{\text {th }}$ thoracic (T13) vertebra (C: one side, D; both sides). In (E), the thirteenth rib on T13 was degenerated.

pair of ectopic ribs on one or both side of L1 spinal column indicating an L1 to T13 transformation. In addition, 1 embryo (6\%) showed less developed T13 ribs. The ectopic L1 ribs were shorter than T13 ribs and varied from a stumplike growth to a pair of bony appendages with cartilaginous ends (Fig. 1C and D). The upper thoracic ribs of these samples were similar to that of control group embryos. Deformed T13 ribs were shorter in length compared to those of control group embryos (Fig. 1E). In this embryo, upper ribs such as T10, 11 and 12 also seemed to be less developed, as their costal cartilages were shorter and less curved around the chest cavity than those of control group embryos. There was no sex preference on these phenotypes.

\section{Effect of prenatal DEX treatment on the protrusion of xiphoid process}

The xiphoid process, indicated in Fig. 2A, is a small cartilaginous process (extension) of the lower part of the sternum. Examination of the sternum have revealed that the xiphoid process in embryos from experimental groups was bent more outward or inward compared to those of control group (Fig. 2B-D and Fig. 3). The y-axis presented
Table 1. Axial skeletal abnormalities found in embryos exposed to prenatal DEX or saline

\begin{tabular}{llr}
\hline \hline Treatments & \multicolumn{2}{c}{ Axial skeletal malformation } \\
\hline Saline $(\mathrm{n}=18)$ & L1 to T13 $(\mathrm{n}=2)$ & $11 \%$ \\
DEX1 $(\mathrm{n}=18)$ & None & $0 \%$ \\
DEX10 $(\mathrm{n}=18)$ & L1 to T13 $(\mathrm{n}=6)$ & $33 \%$ \\
& Abnormal T13 $(\mathrm{n}=1)$ & $6 \%$ \\
\hline
\end{tabular}

in Fig. 3 was an angle between the sternum and the protruding end of the xiphoid process in each embryo. The individuals belonging to the upper outside from the strong dotted line (Mean + SD) in the graph were the embryos possessing xiphoid process that points outward from of the rib cage which bent the sternum into a convex shape (Fig. 2C). In contrast, the ones belonging to the lower outside from the weak dotted line (Mean - SD) showed inward pointing xiphoid process that bent the sternum into a concave shape (Fig. 2D). Female embryos in control saline group were distributed almost equally on the outside or within the criteria 'Mean \pm SD'. Whereas, large proportion of female embryos in DEX1 group (8 out of 9 females) 

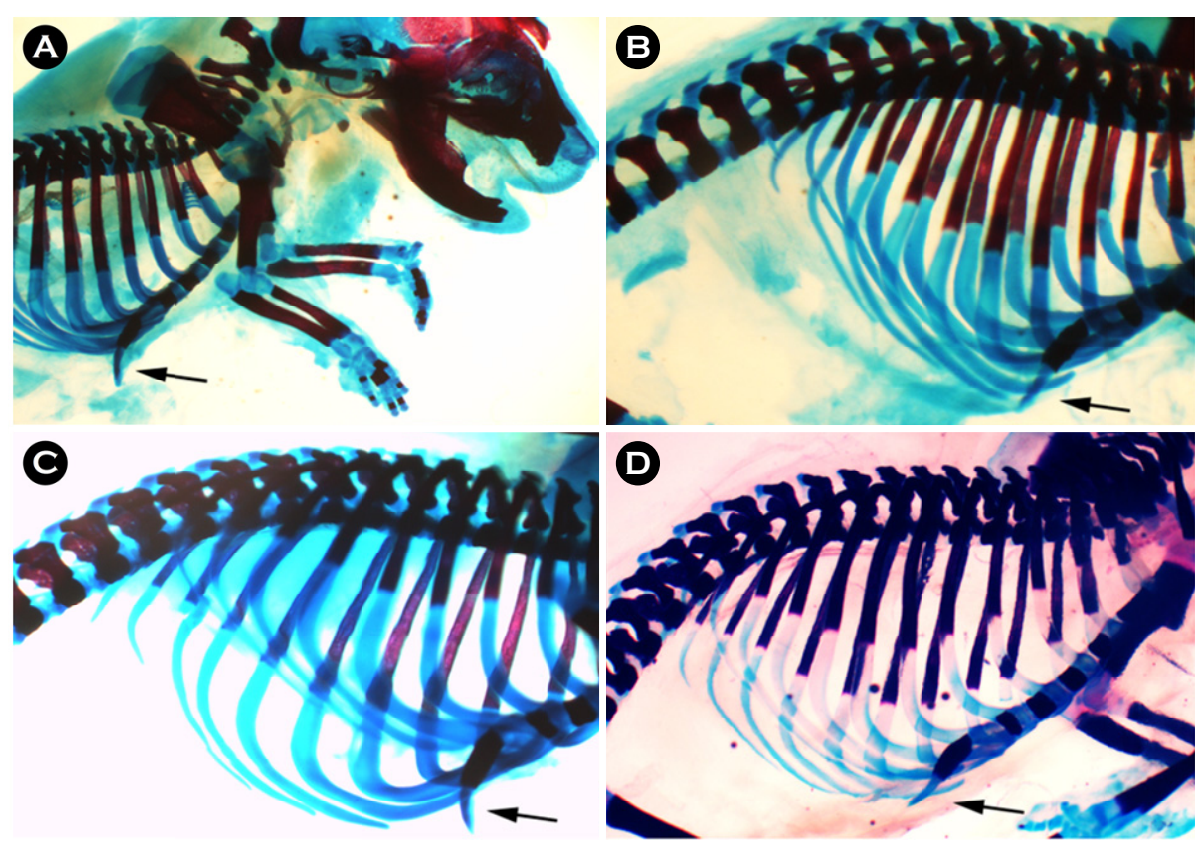

Fig. 2. The xiphoid process of the sternum protruded out from the chest. (A) A typical feature of xiphoid process taken from a saline-treated control embryo. (B) Xiphoid process that belongs to the area of within mean \pm SD. $(\mathrm{C}$ and $\mathrm{D})$ represent xiphoid process that belongs to the area of upper outside and lower outside of mean $\pm \mathrm{SD}$, respectively.

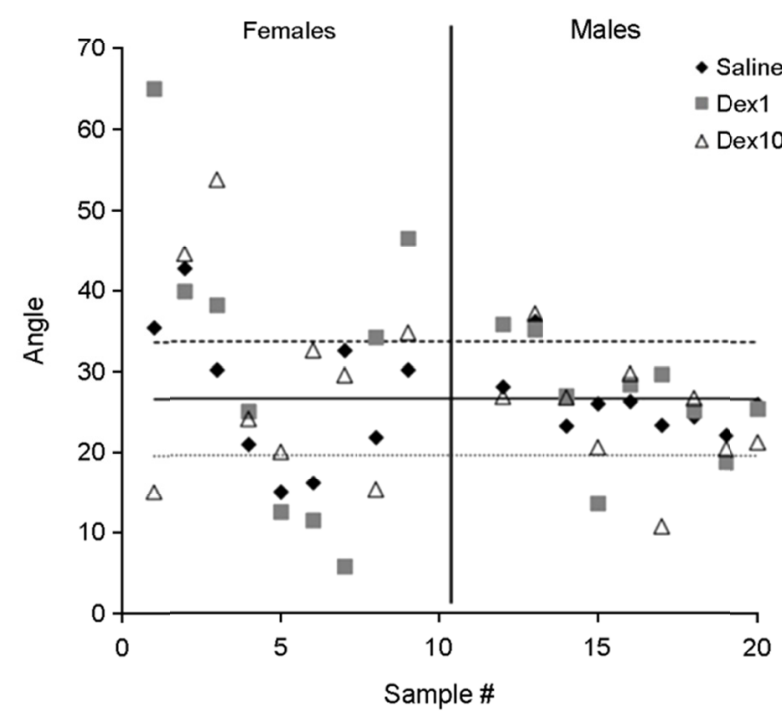

Fig. 3. Xiphoid process protrusion shown in mouse embryos exposed to prenatal DEX. Y-axis represents an angle between long axis of sternum and the edge of xiphoid process. The middle horizontal line is mean value of saline-treated samples. Upper and lower lines indicate mean \pm SD of saline-group samples.

were marked outside of Mean \pm SD, meaning that their xiphoid process were protruded more inward or outward (Table 2). In case of males, only 1 out of 9 males in control group was included in the outside of the criteria, however, the number of males that are included in the outside was increased in DEX1 group (4 out of 9, 44\%). Although the angle formed by the sternum and the end of the xiphoid process was much more variable in females compared to those in males, the xiphoid process of both sexes in DEX1 group showed a distinct tendency to become more bend inward or curve outward. Unexpectedly, for both males and females, there was no big change in DEX10 group embryos.

\section{DISCUSSION}

In this study, the effect of prenatal DEX exposure on skeletal formation was observed. On gestational day 18.5, the embryos in DEX groups showed abnormalities in the thoracic-lumbar boundary: ectopic rib development in the L1 vertebra, which implied an anterior transformation from the L1 to T13 vertebra, and deformed T13 ribs, which may be due to either retardation of bone growth or posterior transformation from T13 to L1. In addition, the xiphoid process of DEX-exposed embryos showed a tendency to protrude more inward or outward.

Although there have been some controversy about the 
Table 2. Number of embryos belonging to the designated area depending on the angle between the sternum and the protruding end of the xiphoid process

\begin{tabular}{lcccccccc}
\hline \hline & \multicolumn{3}{c}{ Female } & & \multicolumn{3}{c}{ Male } \\
\cline { 2 - 4 } \cline { 6 - 8 } & Saline & Dex1 & Dex10 & & Saline & Dex1 & Dex10 \\
\hline Within Mean \pm SD of saline group & $5(56)$ & $1(11)$ & $6(50)$ & & $8(89)$ & $5(56)$ & $8(67)$ \\
Outside of Mean \pm SD of saline group & $4(44)$ & $8(89)$ & & $6(50)$ & & $1(11)$ & $4(44)$ & $3(33)$ \\
\hline
\end{tabular}

The number in parentheses represents a percentage of embryo of a given population.

role of GC in regulating bone formation, GCs have been implicated in the induction of osteoblast differentiation and maintenance of bone homeostasis on a physiological level (Eijken et al., 2006; Henneicke et al., 2014). However, depending on GC dose and duration of experimental condition, as well as in the clinical use of GC for therapy, excess GC has been frequently caused negative effects, such as bone loss or osteoporosis (Weinstein 2011; Henneicke et al., 2014). Similar to the undesirable results of excess GC on bones during the postnatal and adult stages, prenatal GC may also affect skeletal formation during fetal stages and can be programmed.

Maternal restraint stress or DEX exposure in animal models resulted in vertebral and sternal abnormalities, and the retardation of somite and limb formation (Śliwa et al., 2006; Lee et al., 2008; Choe et al., 2011). Several human studies have demonstrated that prenatal GC treatment led to a transient suppression of the levels of carboxy-terminal propeptide of type I collage (PICP) and carboxy-teminal telopeptide of type I collagen (ICTP), a measure to assess the rate of bone formation and resorptive, respectively, but with no long-term impact (Korakaki et al., 2007; Korakaki et al., 2011). However, interestingly enough, Gale et al. have shown that genetic and/or intrauterine environmental factors influenced the fetal growth trajectory and suggested an evidence that low birth weight was associated with lower adult bone and muscle mass, and the risk of osteoporosis in later life (Gale et al., 2001). Therefore, the effects of prenatal GC or other type of stress on bone formation during fetal development and potential impacts over the whole life need to be investigated by specific details.

The vertebrate axial skeleton is composed of cervical, thoracic, lumbar, sacral and caudal regions, with vertebrae in each region. The whole process of the vertebrae formula, from the somite differentiation to the segmentation and the formation of the axial skeleton, is controlled by a variety of molecules, including transcription factors, like the members of the Hox and $C d x$ gene family, and signaling molecules, such as Gdf11, FGF, retinoid acid (RA) or WNT signaling (Mallo et al., 2009). Although there has been no report showing direct connections between the genesis of segmental identity and the GC signaling, GC may affect in the developmental process of axial skeleton formation by affecting other signaling pathways or regulating expression of transcription factors. An evidence showing functional interaction between GC and RA signaling and the resulting effect on Hox gene expression has been demonstrated (Subramaniam et al., 2003). Furthermore, several Hox genes, such as Hoxa5, a9, and $c 10$, has been proposed as GC-regulated genes, in mouse skin (Donet et al., 2008). We have been previously demonstrated that prenatal DEX exposure induces dysregulation of Hox gene expression in mouse embryos (Kim et al., 2011). The similarities of the prenatal DEX-induced phenotypes on skeleton observed in this study, e.g. an ectopic ribs on the L1 vertebrae, with those found in mutants for the Hox paralogous groups 8-10 (Favier and Dolle 1997; van Den Akker et al., 2001; Wellik and Capecchi 2003) suggest possible molecular mechanisms that underlie the detrimental effects of prenatal DEX on skeletal development. Although there were 2 embryos showing supernumerary ribs on the L1 in control group, natural occurrence of the homeotic transformation have been also found at low frequencies within a wild-type population in other studies (del Mar Lorente et al., 2000; van Den Akker et al., 2001; Lorente et al., 2006). Therefore, we do not think that saline injection only caused significant changes in axial skeleton. 
Xiphoid process is a small cartilaginous extension of the lower part of the sternum which is usually ossified in the adult human. The abnormal forward or inward curvature of xiphoid process are not detrimental to the overall health status, but may contribute to the anatomically significant chest deformities, such as pectus excavatum (PE) and pectus carinatum (PC). Patients with PE and PC deformities may feel uncomfortable strains and are unsatisfied with a cosmetic deformity; however, depending on severity, those can impair cardiac and pulmonary function that will be considered for surgical correction (Fokin et al., 2009; Robicsek et al., 2009). Although these type of abnormalities are congenital, neither the systematic analysis of the genetic and molecular mechanisms nor the effects of the early uterine environment have been identified. Based on our observation, the angle divergence between the sternum and the protruding end of the xiphoid process was bigger in female embryos than in male embryos. Sex differences in the effect of prenatal glucocorticoid exposure or stress have now been issued in this field (Bale 2011; Glover and Hill 2012). By the time, the focus of investigation was mostly on sex specific differences in placental function (Cuffe et al., 2011; O'Connell et al., 2011) or sex-biased hypothalamicpituitary-adrenal (HPA) axis activities (Mueller and Bale 2008; Garcia-Caceres et al., 2010), but there was no report about sex differences in the effect of prenatal stress on the skeletal development in fetuses. Our results showing a sex-biased variation in the protrusion of xiphoid process by prenatal DEX exposure could have important implications for the causative effect of uterine environment to the skeletal deformities.

Unexpectedly, it was found that administration of high dose of DEX showed less angle change than the low dose of DEX did, as shown in Table 2 and Fig. 3. In a similar way, embryonic weight and size were less affected by prenatal administration of DEX10 than DEX1 (Yun et al., 2014). Although it is not clear how embryos strive for survival, there should be some complementary mechanisms to overcome unfavorable intrauterine conditions, which could be one reason for less effect with high dose of DEX. We have already demonstrated that a subgroup of placentas from DEX10-injected mother was enlarged with structural abnormalities (Lee et al., 2012). Placentomegaly has been also observed in maternally stressed animal models and human, and therefore, it was regarded as a complementary survival strategy to adapt to an adverse condition (Faichney and White 1987; Lumey 1998; McCrabb et al., 1992; Tegethoff et al., 2010).

In summary, our results demonstrate that prenatal exposure to DEX in mice induces fetal skeletal malformations. Although all of the observable skeletal phenotypes in prenatal stress models of previously published studies are not identical, it should be determined by the timing and the amount of fetal exposure to stress, and by the genetic susceptibility. Therefore, all outcomes from individual studies might provide valuable information and understanding how prenatal maternal stress can affect a developing fetus. Further investigations on the molecular basis behind the phenotypes would be helpful to evaluate the effects on fetal skeletal system.

\section{Acknowledgements}

This research was supported by the Basic Science Research Program (NRF-2010-0025149) through the National Research Foundation (NRF) and partly by a Faculty Research Grant (6-2014-0147) from Yonsei University College of Medicine, Korea.

\section{REFERENCES}

Bale TL. Sex differences in prenatal epigenetic programming of stress pathways. Stress. 2011. 14: 348-356.

Canalis E. Mechanisms of glucocorticoid action in bone. Curr Osteoporos Rep. 2005. 3: 98-102.

Choe HK, Son GH, Chung S, Kim M, Sun W, Kim H, Geum D, Kim K. Maternal stress retards fetal development in mice with transcriptome-wide impact on gene expression profiles of the limb. Stress. 2011. 14: 194-204.

Cuffe JSM, Dickinson H, Simmons DG, Moritz KM. Sex specific changes in placental growth and MAPK following short term maternal dexamethasone exposure in the mouse. Placenta. 2011. 32: 981-989.

del Mar Lorente M, Marcos-Gutierrez C, Perez C, Schoorlemmer J, Ramirez A, Magin T, Vidal M. Loss- and gain-of-function mutations show a polycomb group function for Ring1A in 
mice. Development. 2000. 127: 5093-5100.

Donet E, Bayo P, Calvo E, Labrie F, Perez P. Identification of novel glucocorticoid receptor-regulated genes involved in epidermal homeostasis and hair follicle differentiation. J Steroid Bioche Mol Biol. 2008. 108: 8-16.

Eijken M, Koedam M, van Driel M, Buurman CJ, Pols HA, van Leeuwen JP. The essential role of glucocorticoids for proper human osteoblast differentiation and matrix mineralization. Mol Cell Endocrinol. 2006. 248: 87-93.

Faichney GJ, White GA. Effect of maternal nutritional status on fetal and placental growth and on fetal urea synthesis in sheep. Aus J Biol Sci. 1987. 40: 365-378.

Favier B, Dolle P. Developmental functions of mammalian Hox genes. Mol Hum Reprod. 1997. 3: 115-131.

Fokin AA, Steuerwald NM, Ahrens WA, Allen KE. Anatomical, histologic, and genetic characteristics of congenital chest wall deformities. Semin Thorac Cardiovasc Surg. 2009. 21: 44-57.

Gale CR, Martyn CN, Kellingray S, Eastell R, Cooper C. Intrauterine programming of adult body composition. J Clin Endocrinol Metab. 2001. 86: 267-272.

Garcia-Caceres C, Lagunas N, Calmarza-Font I, Azcoitia I, Diz-Chaves Y, Garcia-Segura LM, Baquedano E, Frago LM, Argente J, Chowen JA. Gender differences the long-term effects of chronic prenatal stress on the HPA axis and hypothalamic structure in rats. Psychoneuroendocrinology. 2010. 35: 1525-1535.

Glover V, Hill J. Sex differences in the programming effects of prenatal stress on psychopathology and stress responses: An evolutionary perspective. Physiol Behav. 2012. 106: 736-740.

Henneicke H, Gasparini SJ, Brennan-Speranza TC, Zhou H, Seibel MJ. Glucocorticoids and bone: local effects and systemic implications. Trends Endocrinol Metab. 2014. 25: 197-211.

Kim SH, Lee J-Y, Park SJ, Kim MH. Effects of dexamethasone on embryo development and Hox gene expression patterns in mice. J Exp Biomed Sci. 2011. 17: 231-238.

Korakaki E, Damilakis J, Gourgiotis D, Katonis P, Aligizakis A, Yachnakis E, Stratakis J, Manoura A, Hatzidaki E, Saitakis E, Giannakopoulou C. Quantitative ultrasound measurements in premature infants at 1 year of age: the effects of antenatal administered corticosteroids. Calcif Tissue Int. 2011. 88: 215 -222 .

Korakaki E, Gourgiotis D, Aligizakis A, Manoura A, Hatzidaki E, Giahnakis E, Marmarinos A, Kalmanti M, Giannakopoulou C. Levels of bone collagen markers in preterm infants: relation to antenatal glucocorticoid treatment. J Bone Miner Metab.
2007. 25: 172-178.

Lee J-Y, Park SJ, Kim SH, Kim MH. Prenatal administration of dexamethasone during early pregnancy negatively affects placental development and function in mice. J Anim Sci. 2012. 90: 4846-4856.

Lee YE, Byun SK, Shin S, Jang JY, Choi B-I, Park D, Jeon JH, Nahm S-S, Kang J-K, Hwang S-Y, Kim J-C, Kim Y-B. Effect of maternal restraint rtress on fetal development of ICR mice. Exp Anim. 2008. 57: 19-25.

Lorente M, Pérez C, Sánchez C, Donohoe M, Shi Y, Vidal M. Homeotic transformations of the axial skeleton of YY1 mutant mice and genetic interaction with the Polycomb group gene Ring1/Ring1A. Mech Dev. 2006. 123: 312-320.

Lumey L. Compensatory placental growth after restricted maternal nutrition in early pregnancy. Placenta. 1998. 19: 105-111.

Mallo M, Vinagre T, Carapuço M. The road to the vertebral formula. Int J Dev Biol. 2009. 53: 1469-1481.

McCrabb GJ, Egan AR, Hosking BJ. Maternal undernutrition during mid-pregnancy in sheep: Variable effects on placental growth. J Agric Sci. 1992. 118: 127-132.

Mueller BR, Bale TL. Sex-specific programming of offspring emotionality after stress early in pregnancy. J Neurosci. 2008. 28: 9055-9065

Newnham JP, Moss TJ. Antenatal glucocorticoids and growth: single versus multiple doses in animal and human studies. Semin Neonatol. 2001. 6: 285-292.

O'Connell BA, Moritz KM, Roberts CT, Walker DW, Dickinson $\mathrm{H}$. The placental response to excess maternal glucocorticoid exposure differs between the male and female conceptus in spiny mice. Biol Reprod. 2011. 85: 1040-1047.

Robicsek F, Watts LT, Fokin AA. Surgical repair of pectus excavatum and carinatum. Semin Thorac Cardiovasc Surg. 2009. 21: 64-75.

Śliwa E, Tatara MR, Nowakowski H, Pierzynowski SG, Studziński T. Effect of maternal dexamethasone and alpha-ketoglutarate administration on skeletal development during the last three weeks of prenatal life in pigs. J Matern Fetal Neonatal Med. 2006. 19: 489-493.

Sloboda D, Challis J, Moss T, Newnham J. Synthetic glucocorticoids: antenatal administration and long-term implications. Curr Pharm Des. 2005. 11: 1459-1472.

Subramaniam N, Campión J, Rafter I, Okret S. Cross-talk between glucocorticoid and retinoic acid signals involving glucocorticoid receptor interaction with the homoeodomain protein Pbx1. Biochem J. 2003. 370: 1087-1095. 
Swolin-Eide D, Dahlgren J, Nilsson C, Albertsson Wikland K, Holmang A, Ohlsson C. Affected skeletal growth but normal bone mineralization in rat offspring after prenatal dexamethasone exposure. J Endocrinol. 2002. 174: 411-418.

Tegethoff M, Greene N, Olsen J, Meyer AH, Meinlschmidt G. Maternal psychosocial stress during pregnancy and placental weight: Evidence from a national cohort study. PLoS One. 2010. 5: e14478.

van Den Akker E, Fromental-Ramain C, de Graaff W, Le Mouellic H, Brulet P, Chambon P, Deschamps J. Axial skeletal patterning in mice lacking all paralogous group 8 Hox genes.
Development. 2001. 128: 1911-1921.

Ward R. Pharmacologic enhancement of fetal lung maturation. Clin Perinatol. 1994. 21: 523-542.

Weinstein RS. Glucocorticoid-induced bone disease. New Eng J Med. 2011. 365: 62-70.

Wellik DM, Capecchi MR. Hox10 and Hox11 genes are required to globally pattern the mammalian skeleton. Science. 2003. 301: 363-367.

Yun HJ, Lee J-Y, Kim J, Kim MH. Effect of prenatal dexamethasone on sex-specific changes in embryonic and placental growth. Biomed Sci Lett. 2014. 20: 43-47. 